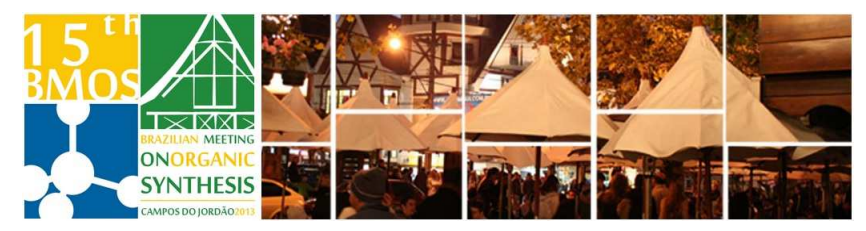

\title{
Effects on Circular Dicroism of pyridil-oxazolinic monomers: preparatory studies on the helical polymer synthesis
}

\author{
George H. Sakae, Rafaela C. Carmona, Edison P. Wendler, Alcindo A. Dos Santos* \\ University of São Paulo, Institute of Chemistry, Av. Prof. Lineu Prestes, 748, São Paulo, SP, Brazil. \\ *alcindo@iq.usp.br.
}

Keywords: 2-oxazolines, chiral monomers, Circular Dicroism

\section{INTRODUCTION}

2-oxazolines are known for a long time but only recently studies concerning their use as catalyst for asymmetric synthesis have gained enormous attention.

The synthesis of copolymers involving 2-oxazoline ring-monomers is known. Functionalized 2oxazoline-homopolymers presents different medicinal and biological applications. ${ }^{2}$

In this study we present the synthesis of optically active alkynyl-pyridyl-oxazoline monomers to be used as precursors of helical polymers. Depending on the nature of the substituent attached to the arylacetylenic unity helical preferentially oriented ( $P$ or M) polymers can be obtained. ${ }^{3}$ These kind of helical polymers may be employed in the molecular and/or ion recognition. ${ }^{4,5}$ Moreover the polymers containing the pyridyl-oxazoline unity will be employed for metal complexation and the resulting organometallics will be explored for recoverable catalysts.

\section{RESULTS AND DISCUSSION}

As an example of the designed monomers we present below the strategy adopted to prepare such compound. p-bromo-cyano pyridine was subject to Pd-catalysed coupling reaction with TMS-acetylene then the corresponding product was converted into the corresponding oxazoline as presented in Scheme 1.
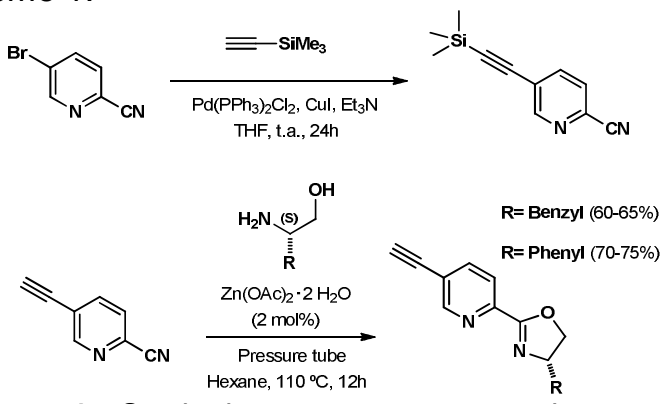

Scheme 1. Synthetic route to prepare the oxazoline monomers

The resulting pyridyl-oxazoline obtained from phenylglycinol was characterized by conventional analytical techniques and submitted to CD on the presence of CuBr-DMS and an amplification of the spectra was observed suggesting a complexation of the cation by both nitrogen atoms. ${ }^{6}$

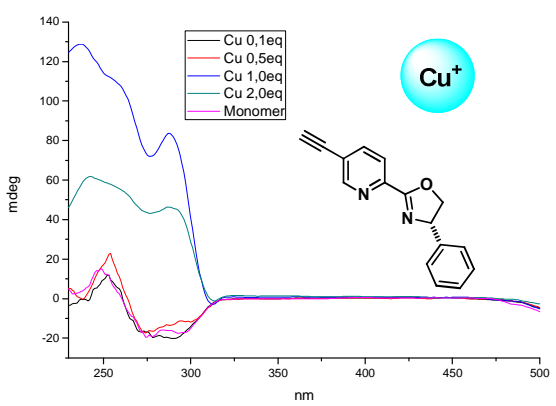

Figure 2. $C D$ of the monomer only and in the presence of CuBr.DMS

Conformer energy calculations were performed for the monomer in absence and presence of copper cations. It was found a $30 \mathrm{~kJ}^{-\mathrm{mol}^{-1}}$ in favor of the syn- $\mathrm{N}, \mathrm{N}$-conformer in detriment of the syn- $\mathrm{N}, \mathrm{O}$ conformer. This kind of behavior can direct helical induction of the resulting polymer.

\section{CONCLUSION}

Beside preliminary, the studies under investigation are promissory specially for a new concept involving external induction of helicity governed by a metal complexation. It is known that a preferential helical orientation can be induced by the bulk of the pendant of the polymer. If the pendant can interacts with an external helical inversion, we can manipulate the supramolecular properties of such polymer and this phenomenon can be used for many purposes including chiral catalysis.

\section{ACKNOWLEDGEMENTS}

The authors are also grateful for the financial and structural support offered by the University of São Paulo through the NAP-CatSinQ (Research Core in Catalysis and Chemical Synthesis), FAPESP, CAPES and CNPq for financial support.

\section{REFERENCES}

${ }^{1}$ Oliveira, A. R. M., et al., Química Nova 1999, 22, 854

${ }^{2}$ Adams, N., Schubert, U. S., Adv. Drug Deliv. Rev, 2007, 59, 1504

${ }^{3}$ Yashima, E. et al., Chem. Commun. 2007, 2351.

${ }^{4}$ Yashima E., et al., J. Am. Chem Soc. 2007, 129, 3676

${ }^{5}$ Riguera R., et al., Angew. Chem. Int. Ed. 2011, 50,11692.

${ }^{6}$ Yashima, E., Goto, H., Okamoto, Y., Macromolecules 1999, 32, 7942 